effects/complications, observations or bloods and collated case vignettes.

Results Between April 2020-Feb 2021, 28 patients were assessed, median age 87.5(43-97) years, 20(71\%) female. Place of care: $13(46 \%)$ own home, the remaining 15 (54\%) resided in care homes/extra care housing. Diagnoses: 19 dementia; 6 cancer, 2 heart failure, 1 Motor Neurone Disease. Frailty scores (Rockwood): median 7(6-9).

$17(61 \%)$ received SCF. Equipment was sourced equally from hospice and local pharmacy. Four had clear benefit, symptom relief, admission avoidance, alive $>1 \mathrm{mth}$ post-intervention. Six cases demonstrated psychological benefit for patient/family with no harm/side-effects (all died 4-17 days post-intervention). Two had possible benefit but diuretics were withheld/reduced concurrently (1 alive $>1$ mth post intervention, 1 died $3 \mathrm{wks})$.

Five had no clear benefit, but no harm. 6/11 cases where SCF were not given, died within a week (2-8 days). Five with dementia in care home/extra-care housing were managed with mouthcare/encouraging oral fluids $(3$ alive $>1$ month postassessment).

Fluids were stopped due to side effects in three patients: none experienced ongoing harm. Volumes of fluid given varied. Feedback from clinicians and families suggested the tool facilitated discussion/understanding of rationale for giving/ withholding.

Conclusion SCF was of clinical benefit for some patients, with minimal harm. The tool increased clinicians' confidence to assess benefits/harms of SCF and address family concerns in a structured way. Further robust data collection is needed to monitor use/outcomes of SCF in a community setting and to better identify patients who may benefit.

\section{P-133 SUBCUTANEOUS FUROSEMIDE USE IN THE COMMUNITY SETTING: CLINICAL BENEFIT AND HOSPITAL AVOIDANCE}

Isobel Jackson, Bruna Burmeister, Fiona Hodson. St Christopher's Hospice, London, UK

10.1136/spcare-2021-Hospice.150

Background There is little evidence on the use of subcutaneous furosemide infusions in heart failure patients, especially in the community setting (Beattie \& Johnson, 2012). Integrated working is key to smooth the transition from management in the hospital to community settings, particularly for patients who are increasingly fatigued with short prognoses but who may still benefit from parenteral therapy.

Aim To retrospectively evaluate clinical effectiveness of subcutaneous furosemide used in the community setting at end-oflife.

Method We reviewed case notes of patients treated with continuous subcutaneous furosemide by the community palliative care team (2019-2021). A standardised proforma was used to assess patient demographics, indications, clinical outcomes and barriers/facilitators to the process.

Results 16 patients received a total 22 interventions. Median age $77(50-94), 11(69 \%)$ male, primary HF (Heart failure) diagnosis 6 HFrEF, 5 HFpEF,2 RHF, 2 Valvular, 1 unknown aetiology.

Phase of Illness, 13 unstable and 9 deteriorating with AKPS 30-60\%. Oral diuretics included loop diuretics (furosemide 15/ 22 and bumetanide 6/22), aldosterone receptor antagonists (spironolactone 4/22), mineralocorticoid receptor antagonists (eplerenone 11/22) and thiazides (indapamide 1/22). Majority of interventions had stage 3 renal function (16/22 episodes). Patients were discussed with cardiology or palliative care consultant. Median dose given was $160 \mathrm{mg}$ (range $80-240 \mathrm{mg}$ ) for 11(1-33) days.

Outcomes 15/22 showed symptomatic improvement, 6/22 had side-effects including worsening renal function, hypotension, hypokalaemia and site reaction needing antibiotics. 21/22 interventions avoided a hospital admission; 12/16 patients achieved preferred place of death (1 was admitted to hospital; 3 alive). Barriers to community administration included: challenges obtaining medications, burden of a continuous sub cutaneous infusion [CSCI].

Conclusion There is a clear benefit for individual patients, allowing those in last year of life to spend as much time as possible at home. We plan to develop a decision support tool to aid wider community decision making, facilitating timely use of subcutaneous furosemide, therefore maintaining effective symptom control in community settings.

\section{P-134 SYMPTOMS MATTER; AN AUDIT OF SYMPTOMATIC MANAGEMENT OF MALIGNANT BOWEL OBSTRUCTION}

Oliver Emmerson. Pennine Acute NHS Trust, Manchester, UK

10.1136/spcare-2021-Hospice. 151

Background The diagnosis, assessment and management of malignant bowel obstruction (MBO) varies across organisations. Different levels of emphasis are placed on resolution of the obstruction or symptomatic management. Yet there is currently a lack of comprehensive guidance or clinical pathways to ensure an equivalent high-standard level of care is offered to all patients.

Aims

- To determine current practice in the management of MBO.

- To inform future guidance and direct the emphasis placed on specific management strategies.

- To improve future patient care and experience through more effective symptomatic management.

Methods Patients with an inpatient diagnosis of MBO were identified from the specialist palliative care (SPC) multi-disciplinary [MDT] lists from March 2019-January 2020. Data collected included: demographics, admission length/outcome, diagnosis, symptomatic reviews and treatment methods. Data was tabulated and analysed in Microsoft Excel.

Results Fifteen inpatients were identified. Seven patients (47\%) died in hospital on average 22 days after admission. Nine $(60 \%)$ had known colorectal malignancies. Fifteen $(100 \%)$ had nausea and vomiting or colic on admission. Of the nine (60\%) who had colic on admission five (34\%) still had colic on day 4.

Only five $(45 \%)$ had a daily review of symptoms. By day four, 10(67\%) had PRN anti-emetics prescribed and 5(34\%) had regular anti-emetics prescribed. The most commonly used agents were cyclizine (PO/IM/IV) and ondansetron (PO/IM/IV). Ten (77\%) had naso-gastric [NG] tubes inserted and none underwent surgical procedures. All were reviewed by the inpatient SPC team at least once during their admission.

Conclusions Surgical management of $\mathrm{MBO}$ is uncommon and so emphasis should be placed on symptomatic relief and conservative therapy options. This would enable greater patient autonomy to decide preferred place of death. Assessment of symptoms of $\mathrm{MBO}$ should be clearly documented on 
assessment and daily. Assessment of colic is often missed and prompt commencement of anti-colic therapy would benefit patient care. Standardisation of anti-emetic choice in $\mathrm{MBO}$ would be beneficial, leading to reduced inappropriate prescribing.

\section{P-135 AMENDED MOUTH CARE MATTERS PROJECT}

Jamie Yeomans. St Richard's Hospice, Worcester, UK

10.1136/spcare-2021-Hospice. 152

Background A common problem in palliative care is that of a dry and sore oral environment often resulting in infections, bad breath (halitosis), and changes in taste, therefore high quality mouth care is a very important aspect of palliative care in all care settings. When these problems are not managed effectively, they can negatively affect a patient's selfesteem, ability to communicate and socialise plus the ability to enjoy food and drinks (resulting in inadequate nutrition/hydration). It is vital therefore that mouth problems should be assessed regularly and treated as soon as possible (Marie Curie. Mouthcare, see: mariecurie.org.uk/professionals/palliative-care-knowledge-zone/symptom-control/mouth-care/).

Aims The Mouth Care Matters programme (Health Education England, see: https://mouthcarematters.hee.nhs.uk/) was designed to help deliver better clinical outcomes by evidencing the importance of good mouth care and how this positively impacts on general health and quality of life.

Methods We piloted the original '(Mouthcare Matters) Assessment and Recording Form' in the inpatient unit with the aim of providing a thorough method of assessing and addressing oral challenges experienced by our palliative/end-of-life patients (Venkatasalu, Murang, Ramasamy, et al., 2020). We then audited outcomes by publishing a scored staff feedback form to colleagues to measure effectiveness of the intervention and explore whether amendments to the tool could be implemented to make it more bespoke to our care setting (National Institute for Health and Care Excellence: Palliative care: oral. See: cks.nice.org.uk/topics/palliative-care-oral).

Results When feedback was submitted, qualitative evidence identified the need to amend the standard to truly fit the hospice model of care.

Conclusions We amended the template in the following ways:

- The addition of 'saliva' as an identifiable problem (Paine \& Snider, 2020).

- Changed 'weekly' assessment to 'daily' to reflect the pace of change some of our patients experience.

- The original tool identified low, medium and high risks but there was no advice to prompt intervention so we added an 'action checklist' section to help direct care.

We launched this amended version in April 2021 and are due to audit results in September 2021.

\section{P-136 FOUR STAGE LOW FIBRE DIETARY GUIDANCE FOR PATIENTS SUFFERING SUBACUTE MALIGNANT BOWEL OBSTRUCTION}

Sarah Onions, Nicola Wilderspin. St Richard's Hospice, Worcester, UK

10.1136/spcare-2021-Hospice.153
Background Patients with subacute malignant bowel obstruction (MBO) not amenable to surgical or interventional procedures are often highly symptomatic of pain, bloating, nausea and vomiting. Diet often exacerbates symptoms so intake is limited. UK medical treatment of $\mathrm{MBO}$ includes medicines and interventions to improve bowel transit and to manage symptoms (Ahmad, Jeffries, Longford, et al., 2015; Scottish Palliative Care Guidelines, 2020) but often little specific advice is given on low fibre diet compared to approaches worldwide (Lee, Jivraj, Wang, et al., 2019; British Columbia Cancer Agency, 2019).

Aim To develop a low fibre staged diet plan for patients with subacute or resolving MBO. To offer patients choice and taste variety within the limitations of a clear fluid or low residue diet in order to improve symptom control, chance of resolution and quality of life.

Methods A review of literature was undertaken to better understand diet in MBO. A UK hospital's oncology dietician team use a phased model on which, with permissions, our diet is based. A collaborative approach was taken including input from the local trust dieticians and hospice multi-disciplinary team including catering and hospitality.

Results A four staged diet plan was created for our inpatient unit. Stage one, clear fluids; Stage two, liquid low fibre; Stage three, soft low fibre; Stage four, normal texture low fibre. MBO patients often start with bowel rest and move up or down the stages as tolerated. Stage four may also be used prophylactically for those at risk of developing MBO symptoms. Conclusion Most patients have welcomed specific guidance on diet. The menu provides variety despite the restrictions and many patients report significant relief of their symptoms. Patients may choose to eat off plan, as part of our 'Eat for Comfort' policy. A community MBO diet advice booklet has subsequently been created for those living with or at risk of $\mathrm{MBO}$, enabling patients to stay at home longer and be more in control of their symptoms.

\section{P-137 VIRTUAL REALITY IN PALLIATIVE CARE}

Jenny Warren, James Elliott, Clare Mills. Compton Care, Wolverhampton, West Midlands

\subsection{6/spcare-2021-Hospice.154}

Introduction COVID-19 pushed us as an organisation to stepup our ability to change and adapt. Our workforce has embraced the opportunities generated by digital transformations during the pandemic. Virtual Reality (VR) is revolutionising healthcare. We wanted to explore the use of VR as part of a non-pharmacological approach to symptom management and start to measure patient outcomes in a meaningful way while improving the patient's overall experience.

Actions Our initial proposal was that the use of the VR headset could positively impact on patient wellbeing. Our living well centre team commenced a pilot in conjunction with our inpatient unit. As new technology becomes more accessible use of these as part of the patient's overall plan of care is a realistic possibility even for small organisations.

Utilising freely available innovative apps the team supported patients in immersive experiences including guided relaxation meditation and visits to locations such as white sandy beaches, forests and mountain ranges. Initial outcomes were positive utilising a simple wellbeing score and numerical pain scoring. This has led us, in conjunction with a digital fabrication laboratory based at a local college, to look at 